\title{
Разработка математической модели для оптимизации конструкции автомобильного термоэлектрического генератора с учетом влияния его гидравлического сопротивления на мощность двигателя
}

\author{
(ㄱ Р.А. Пошехонов, Г.А. Арутюнян, С.А. Панкратов, А.С. Осипков ", Д.О. Онищенко, А.И. Леонтьев
}

Московский государственный технический университет им. Н.Э. Баумана

(Национальный исследовательский университет),

105005 Москва, Россия

๑E-mail: osipkov@bmstu.ru

(Получена 31 января 2017 г. Принята к печати 8 фревраля 2017 г.)

\begin{abstract}
Предложен подход и разработан комплекс моделей для оптимизации конструкции и режимов работы автомобильного термоэлектрического генератора с учетом влияния его гидравлического сопротивления на двигатель внутреннего сгорания. Рассмотрен ряд конструкций генераторов, предназначенных для преобразования в электричество тепловой энергии отработавших газов двигателя внутреннего сгорания за счет эффекта Зеебека в полупроводниковых элементах, отличающихся геометрией проточной части генератора с различным гидравлическим сопротивлением. Совместно рассмотрены модели для расчета термоэлектрических элементов, газового теплообменника и автомобильного двигателя. Проведено моделирование на примере двигателя ВАЗ 21126, показавшее возможность получения до 500 ВТ электрической мощности при использовании полупроводниковых термоэлектрических элементов на основе теллуридов германия и свинца.
\end{abstract}

DOI: 10.21883/FTP.2017.08.44778.47

\section{1. Введение}

Разработка эффективных конструкций термоэлектрических генераторов (ТЭГ) для автомобилей может открыть широкий рынок для полупроводниковых термоэлектрических батарей (ТЭБ), в основе работы которых лежит эффект Зеебека [1-3]. Для этого на сегодняшний день существует два основных препятствия [3]: во-первых, низкая эффективность промышленно выпускаемых в настоящее время полупроводниковых материалов, приводящая к низкому КПД такого генератора, увеличению его массогабаритных характеристик; во-вторых, трудность конструирования ТЭГ из-за необходимости комплексного рассмотрения тепловых, электрических, аэродинамических, гидравлических процессов.

Разработка новых полупроводниковых термоэлектрических материалов с высокой термоэлектрической добротностью $Z=\sigma \alpha^{2} k^{-1}, \quad(\sigma-$ электропроводность, $\alpha$ - дифференциальная термоэдс, $k-$ теплопроводность) ведется во многих научных центрах [4]. В настоящее время наблюдаются некоторые проблемы с переходом от лабораторных образцов к промышленному производству таких материалов, однако рано или поздно они будут решены. Преодоление второго препятствия требует создания комплексной расчетной модели ТЭГ с целью выбора рациональных конструкторскотехнологических решений при его проектировании, чему и посвящена данная работа.

При проектировании ТЭГ существует конфликт целей из-за необходимости в ограниченных габаритах одновременно обеспечить интенсивный поток тепла через полупроводниковые термоэлектрические элементы и малое газодинамическое сопротивление. В таких конструкциях теплообмен ограничен контактными сопротивлениями и интенсивностью конвекции [3], характеризуемой безразмерным числом Нуссельта $N u$. В прототипах таких конструкций используют турбулизаторы отработавших газов (ОГ), оребрение, лунки и другие элементы, которые вместе с тем увеличивают перепад давления в канале отработавших газов $\Delta p_{\text {gas }}$, что снижает эффективность двигателя.

Моделированию теплоотвода в автомобильных ТЭГ уделено пристальное внимание [2,3,5-9]: рассмотрены конструкции плоских (прямоугольных) [2,3,5] и шестигранных теплообменников с ребрами $[3,5,6]$, интенсификаторами конвективного теплообмена [2] и вытеснителями [6], которые позволяют увеличить число Нуссельта $N u$. В работах $[2,5,6]$ определялся перепад давления ОГ $\Delta p_{\text {gas }}$ в ТЭГ. Вместе с тем в приведенных работах не учтено влияние сопротивления ТЭГ на работу двигателя внутреннего сгорания (ДВС), что не позволяет на основании $\Delta p_{\text {gas }}$ оценить эффективность конструкции ТЭГ.

В настоящей работе предложена методика оценки эффективности ТЭГ с заданным ДВС. Методика позволяет оптимизировать конструкцию ТЭГ с учетом влияния его аэродинамического сопротивления на мощность двигателя.

\section{2. Конструкция и принцип действия ТЭГ}

Рассматриваемая конструкция ТЭГ была подробно описана в работах $[10,11]$. На рис. 1 представлена структурная схема подключения ТЭГ к системам автомобиля, и обозначены математические модели, использующиеся для расчета компонентов ТЭГ. 
Горячие ОГ, двигаясь от ДВС (1), проходят через ТЭГ (2). При этом часть внутренней энергии ОГ отводится в виде теплового потока $Q_{\text {hot }}$ через горячий теплообменник (3), ТЭБ (4) и водяные холодные теплообменники (5). В ТЭБ (4) часть теплого потока $Q_{\text {hot }}$ преобразуется в электрическую мощность $W_{\text {teb }}$ за счет эффекта Зеебека. От ТЭГ тепловой поток может отводиться через собственную или общую автомобильную гидравлическую систему охлаждения с водяным насосом и радиатором. Часть вырабатываемой ТЭБ электрической мощности $W_{\text {teb }}$ расходуется на функционирование собственной или догрузку общей систем охлаждения.

На рис. 1 обозначены усредненные во времени затраты электрической мощности: $W_{\text {ротр }}$ - водяного насоса $(6)$, $W_{\text {rad }}$ - вентилятора (7). Оставшаяся мощность

$$
W_{\text {teg }}=W_{\text {teb }}-W_{\text {pomp }}-W_{\text {rad }}
$$

поступает в бортовую систему автомобиля, благодаря чему может быть разгружен или удален штатный электромеханический генератор $(8)$.

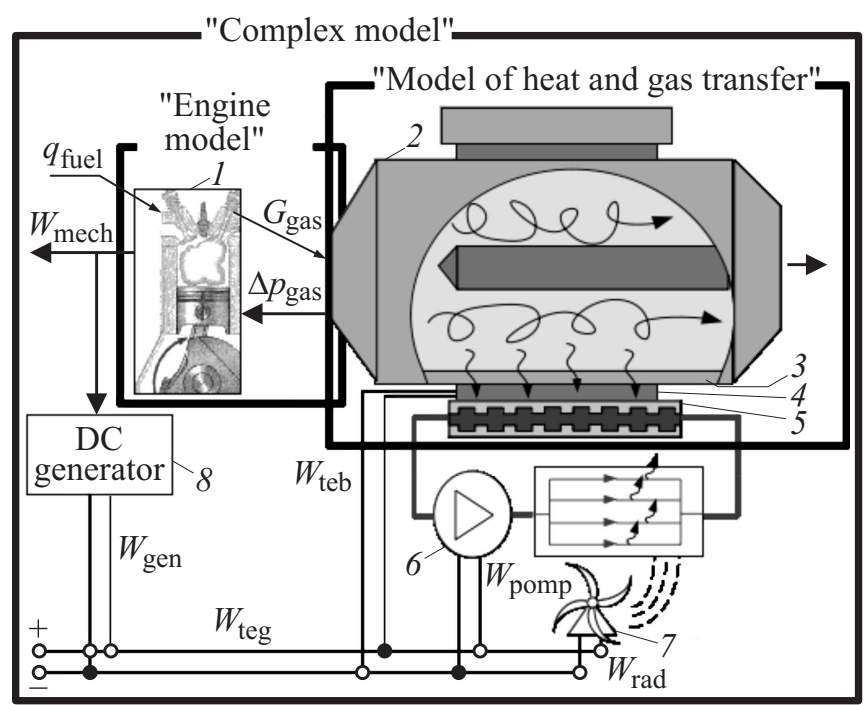

Рис. 1. Структурная схема ТЭГ.

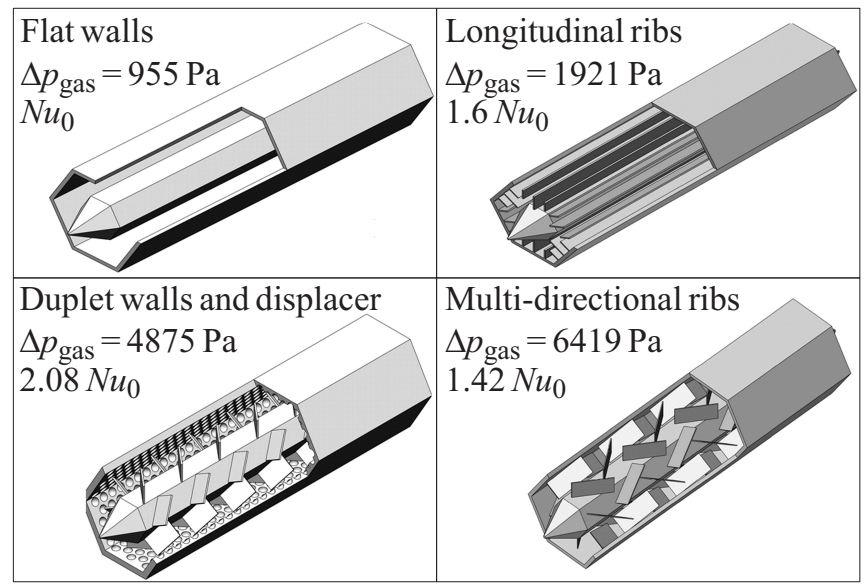

Рис. 2. Рассмотренные конструкции горячего воздушного теплообменника.
Таблица 1. Параметры термоэлектрических полупроводников

\begin{tabular}{c|c}
\hline Коэффициенты термоэдс, $\mathrm{B} \cdot \mathrm{K}^{-1}$ & $\alpha_{n}=1.87 \cdot 10^{-4}$ \\
& $\alpha_{p}=2.25 \cdot 10^{-4}$ \\
\hline Теплопроводности, Вт $\cdot(\mathrm{K} \cdot \mathrm{M})^{-1}$ & $k_{n}=1.4$ \\
& $k_{p}=1.5$ \\
\hline Электропроводности, $(\mathrm{OM} \cdot \mathrm{M})^{-1}$ & $\sigma_{n}=3.23 \cdot 10^{4}$ \\
& $\sigma_{p}=4.67 \cdot 10^{4}$
\end{tabular}

За основу ТЭГ взята шестигранная конструкция теплообменника с водяным охлаждением и центральным вытеснителем, увеличивающим скорость ОГ и интенсивность теплоотдачи [6]. Модификации такого теплообменника с различными типами внутреннего оребрения рассмотрены в работе [10]. В этой работе для режима, соответствующего максимальной мощности ДВС, сопоставлены перепады давления в ТЭГ $\Delta p_{\text {gas }}$ и изменение числа Нуссельта $N u$ по сравнению с теплообменником, имеющим гладкие стенки. Наиболее интересные компоновки представлены на рис. 2 с числами Нуссельта и перепадом давления в ТЭГ.

Термоэлектрические модули имеют площадь $79 \times 57$ мм и толщину 11 мм. Для ТЭГ выбраны полупроводниковые термоэлектрические материалы, освоенные отечественной промышленностью. Ветви $n$-типа изготовлены из теллурида свинца. Сегментные ветви $p$-типа изготовлены из теллурида висмута и теллурида германия и соединены слоем кобальта. Усредненные параметры материалов для рабочего диапазона температур приведены в табл. 1. Для рассмотренных конструкций параметр, характеризующий термоэлектрическую эффективность, критерий Иоффе $Z T$ менялся в диапазоне $0.4 \ldots 1$ в зависимости от материала ( $p$ - или $n$-тип) и температуры.

\section{3. Постановка задачи}

Рассмотренные ТЭГ (рис. 2) имеют одинаковые габариты и толщины стенок теплообменников и ТЭБ, а также свойства термоэлектрических материалов. Поэтому масса и стоимость данных конструкций при серийном изготовлении будут схожи.

Интегральным критерием качества ТЭГ выбрано увеличение механической мощности на валу ДВС

$$
\Delta W_{\text {mech }}=W_{\text {add }}-W_{\text {press }},
$$

где $W_{\text {add }}=W_{\text {teg }} / \eta_{\text {gen }}$ - приращение мощности за счет разгрузки электромеханического генератора, имеющего КПД $\eta_{\text {gen }} ; W_{\text {press }}$ - уменьшение мощности на валу изза дополнительного перепада давления $\Delta p_{\text {gas }}$ в канале движения ОГ.

$W_{\text {add }}$ и $W_{\text {press }}$ сильно зависят от режима течения ОГ внутри ТЭГ, который определяется температурой ОГ и скоростью вращения ДВС $n_{E}$. 


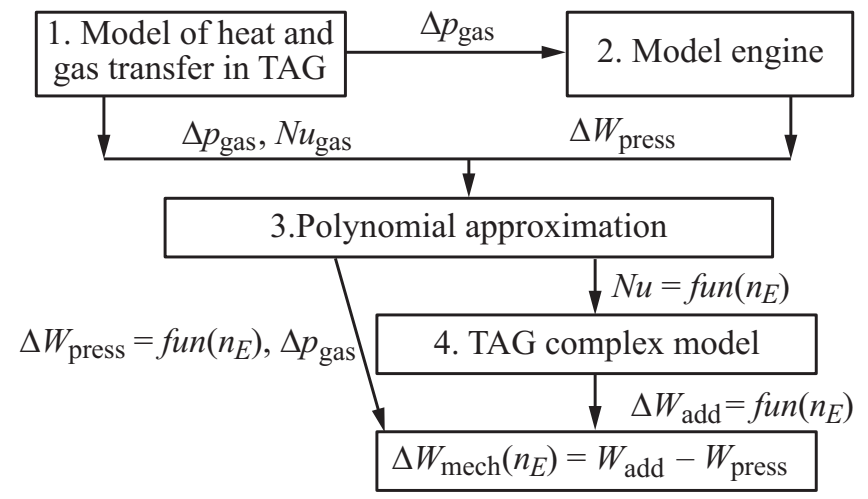

Рис. 3. Последовательность моделирования.

\section{4. Последовательность расчета}

Для расчета $\Delta W_{\text {mech }}$ необходимо описать множество процессов как внутри ТЭГ, так и внутри ДВС, а для этого использованы три расчетные модели. Последовательность моделирования $\Delta W_{\text {mech поясняет рис. } 3 .}$

1. Модель тепло-газодинамики позволяет определить интегральные значения числа Нуссельта $N u_{\text {gas }}$ и перепад давления ОГ в ТЭГ $\Delta p_{\text {gas }}$ для объемного турбулентного потока ОГ $G_{\text {gas }}$, соответствующего максимальной скорости вращения вала ДВС $n_{E \max }$.

2. Модель двигателя позволила определить потерю механической мощности $\Delta W_{\text {mech }}$ на валу ДВС, вызванную увеличением давления ОГ $\Delta p_{\text {gas. }}$.

Данные расчеты весьма трудоемки, поэтому они были выполнены для ряда характерных значений, после чего построены аппроксимационные зависимости для числа Нуссельта $N u_{\text {gas }}$ и перепада давления $\Delta p_{\text {gas }}$ от скорости вращения вала $n_{E}$, а также для потерь механической мощности в ДВС $\Delta W_{\text {mech }}$ от перепада давления $\Delta p_{\text {gas }}$ и скорости вращения вала $n_{E}$.

3. Общая модель ТЭГ [11] позволяет определить $W_{\text {add }}$ в зависимости от скорости вращения $n_{E}$. Ниже приведены пояснения к этапам расчета.

В расчетах рассмотрен бензиновый четырехцилиндровый двигатель ВАЗ 21126 с рабочим объемом $V_{h}=1.6$ л, мощностью $72 \mathrm{\kappa BT} \mathrm{при} n_{E}=5800$ мин $^{-1}$. Основные параметры ТЭГ приведены в статье [11].

4.1. Моделирование тепло-газодинамики внутри ТЭГ проводилось численно методом контрольных объемов в программном комплексе FlowSimulation при использовании уравнений Навье-Стокса с осреднением по Фавру и модифицированной $k-\varepsilon$ модели турбулентности и уравнения теплопроводности Фурье [10]. В результате расчета для разных конструкций проточной части ТЭГ определены перепады давления ОГ $\Delta p_{\text {gas }}$ и числа Нуссельта $N u_{\text {gas }}$ для режима работы ДВС с максимальной скоростью $n_{E}$ и максимальной мощностью (рис. 3).

4.2. Моделирование ДВС проведено в программном комплексе DIESEL-RK [12] на основе нульмерной многозонной математической модели на примере двигателя
BA3 21126 в режиме максимальной мощности. Расчеты выполнены численно для ряда значений перепада давления $\Delta p_{\text {gas }}$ на выходе из ДВС и скорости вращения $n_{E}$. В результате получено падение механической мощности ДВС

$$
W_{\text {press }}\left(n_{E}, \Delta p_{\text {gas }}\right)=W_{\text {mech }}\left(0, n_{E}\right)-W_{\text {mech }}\left(\Delta p_{\text {gas }}, n_{E}\right) .
$$

4.3. Аппроксимация выражения для потери мощности ДВС была выполнена полиномиальной зависимостью второго порядка

$$
W_{\text {press }}\left(n_{E}, \Delta p_{\text {gas }}\right)=\sum_{i=0}^{2} \sum_{j=0}^{2} k_{i, j} n_{E}^{i} \Delta p_{\text {gas }}^{j}
$$

c коэффициентами $k_{0,0}=-4.35 \mathrm{BT}, \quad k_{1,0}=$ $=-0.00182 \mathrm{BT} \cdot \Gamma_{\bigsqcup^{-1}}, \quad k_{0,1}=0.205 \mathrm{BT} \cdot \Pi_{a^{-1}}, \quad k_{1,1}=$ $=-1.27 \cdot 10^{-5} \mathrm{BT} \cdot \Gamma_{L^{-1}}{ }^{-1} \cdot \Pi^{-1}, k_{0,2}=3.33 \cdot 10^{-6}{ }^{\prime} \mathrm{BT} \cdot \Pi^{-1}$.

Частота вращения коленчатого вала ДВС $n_{E}$ прямо пропорциональна усредненной скорости ОГ [13], а также числу Рейнольдса $\mathrm{Re}_{\mathrm{gas}}$, поэтому для потерь давления в ТЭГ $\Delta p_{\text {gas }}$ использована квадратичная зависимость, поскольку течение является турбулентным:

$$
\Delta p_{\text {gas }}\left(n_{E}\right)=\Delta p_{\text {gasgas }}^{\max } \frac{n_{E}^{2}}{n_{\text {gas }}^{\max }},
$$

где $\Delta p_{\text {gas }}^{\max }$ - перепад давления, определенный при моделировании тепло-газодинамики для частоты вращения коленчатого вала, соответствующей максимальной мощности ДВС $n_{\mathrm{gas}}^{\max }$.

Для аппроксимации зависимости числа Нуссульта скорректирован коэффициент длины $\varepsilon_{\text {Leg }}$ в формуле для прямой трубы с турбулентным потоком газа [14]:

$$
\mathrm{Nu}_{\text {gas }}=0.022 \mathrm{Re}_{\text {gas }}^{0.8} \operatorname{Pr}_{\text {gas }}^{0.43} \varepsilon_{L},
$$

где $\mathrm{Pr}_{\text {gas }}$ - число Прандтля для ОГ. В итоге получено выражение для аппроксимации числа Нуссельта

$$
\mathrm{Nu}_{\text {gas }}=\mathrm{Nu}_{\text {gas }}^{\max } \frac{n_{E}^{0.8}}{n_{E}^{\text {max }} .8}
$$

4.4. Общая модель ТЭГ [11] позволяет определить электрическую мощность $W_{\text {teb }}$, вырабатываемую термоэлектрической батареей, а также электрическую мощность $W_{\text {teg }}$, выдаваемую в электрическую сеть автомобиля с учетом затрат энергии на догрузку вентилятора и помпового насоса системы водяного охлаждения. При расчете конвективного теплообмена горячего теплообменника использованы аппроксимационные выражения (7), полученные для рассмотренных компоновок ТЭГ (рис. 3).

По сравнению с работой [11] общая модель ТЭГ дополнена для учета контактных тепловых сопротивлений и изменения механической мощности на валу. Экспериментально определенные значения контактных сопротивлений приведены в табл. 2. Падение мощности 


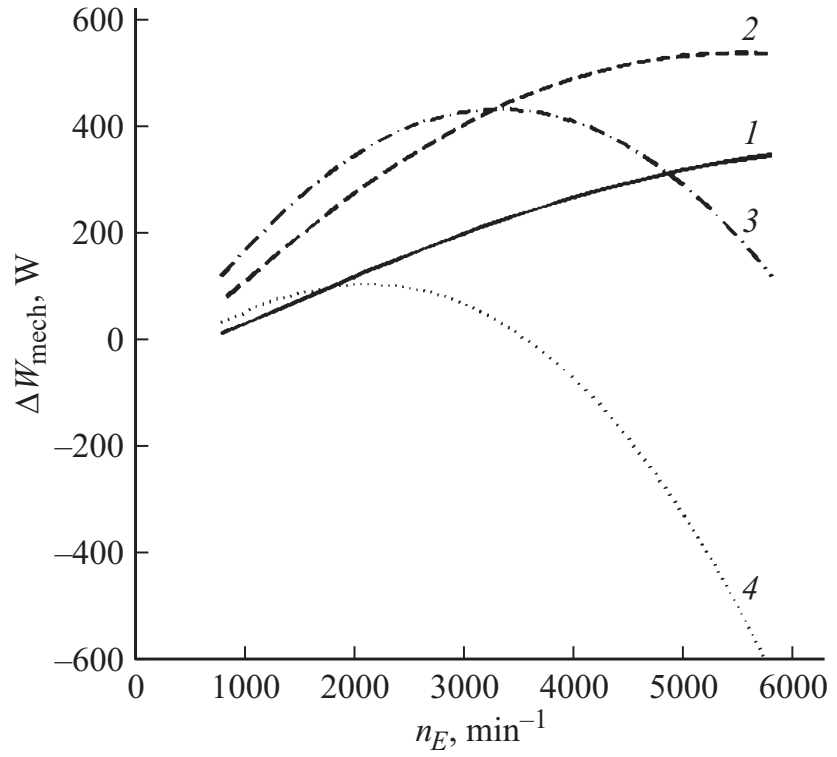

Рис. 4. Суммарное увеличение мощности на валу $\Delta W_{\text {mech }}$ в зависимости от скорости вращения $n_{E}: 1-$ плоские стены, 2 - продольные ребра, 3 - демпфированные стены и буек, 4 - разнонаправленные ребра.

ДВС $\Delta W_{\text {mech }}$ определяется по сравнению с режимом вращения вала при максимальной мощности ДВС со штатным генератором

$$
\Delta W_{\text {mech }}\left(n_{E}\right)=\frac{W_{\text {teg }}\left(n_{E}\right)}{\eta_{\text {gen }}^{-1}}-W_{\text {press }}\left[n_{E}, \Delta p_{\text {gas }}\left(n_{E}\right)\right] \text {. }
$$

При расчетах принят усредненный по времени КПД штатного генератора $\eta_{\mathrm{gen}}=0.5$.

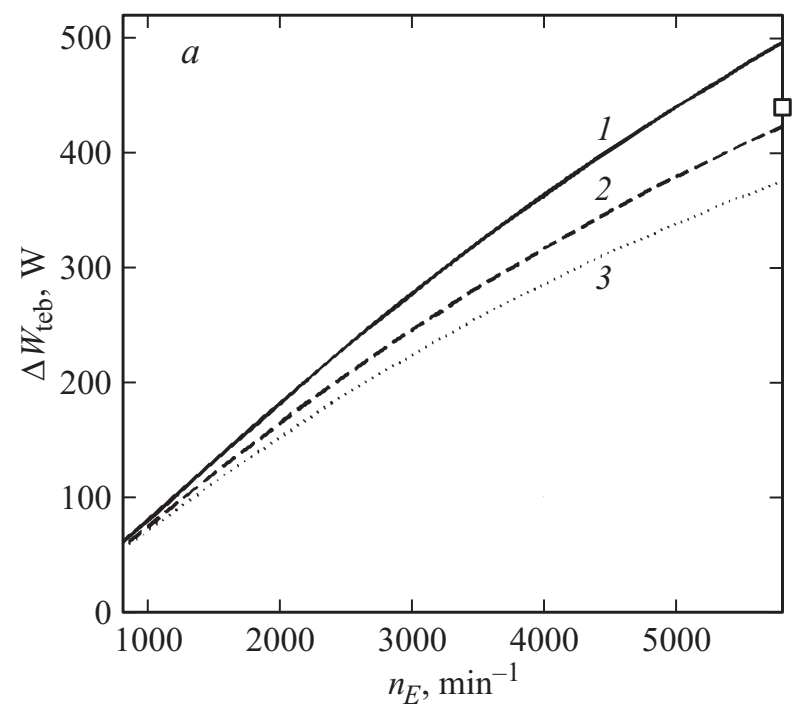

Таблица 2. Коэффициенты удельного контактного сопротивления

\begin{tabular}{c|c|c}
\hline \multirow{2}{*}{$R_{\text {cont_hot, }} \mathrm{K} \cdot \mathrm{M}^{2} \cdot \mathrm{BT}^{-1}$} & Без всего & $9.96 \cdot 10^{-4}$ \\
\cline { 2 - 3 } & $\begin{array}{c}\text { С эмалью } \\
\text { Вместо термопасты }\end{array}$ & $5.67 \cdot 10^{-4}$ \\
\hline \multirow{2}{*}{$R_{\text {cont_cold }}, \mathrm{K} \cdot \mathrm{M}^{2} \cdot \mathrm{BT}^{-1}$} & Без всего & $11.7 \cdot 10^{-4}$ \\
\cline { 2 - 3 } & С термопастой & $6.37 \cdot 10^{-4}$
\end{tabular}

\section{5. Результаты моделирования и обсуждение}

На рис. 4 приведены результирующие зависимости изменения механической мощности $\Delta W_{\text {mech }}$ на валу ДВС от скорости вращения $n_{E}$ для различных конструкций теплообменников с идеальными тепловыми стыками $\left(R_{\text {cont }}=0\right) . \Delta W_{\text {mech }}$ сильно меняется и может быть как положительна, так и отрицательна. При малых скоростях вращения вала лучше всего работает компоновка с лунками и турбулизатором, при больших с продольными ребрами. ТЭГ с разнонаправленными ребрами создает настолько большое аэродинамическое сопротивление, что в результате $\Delta W_{\text {mech }}$ становится отрицательной.

На рис. 5 приведены результаты расчета вырабатываемой термоэлектрической мощности $W_{\text {teb }}$ и увеличения мощности ДВС $\Delta W_{\text {mech }}$ в зависимости от $n_{E}$ для ТЭГ с продольными ребрами и различными тепловыми контактными сопротивлениями $R_{\text {cont }}$. Значения $R_{\text {cont }}$ (табл. 2) оценены экспериментально для сухих шлифованных стыков и стыков, заполненных эмалью и термопастой. Они были оценены экспериментально с

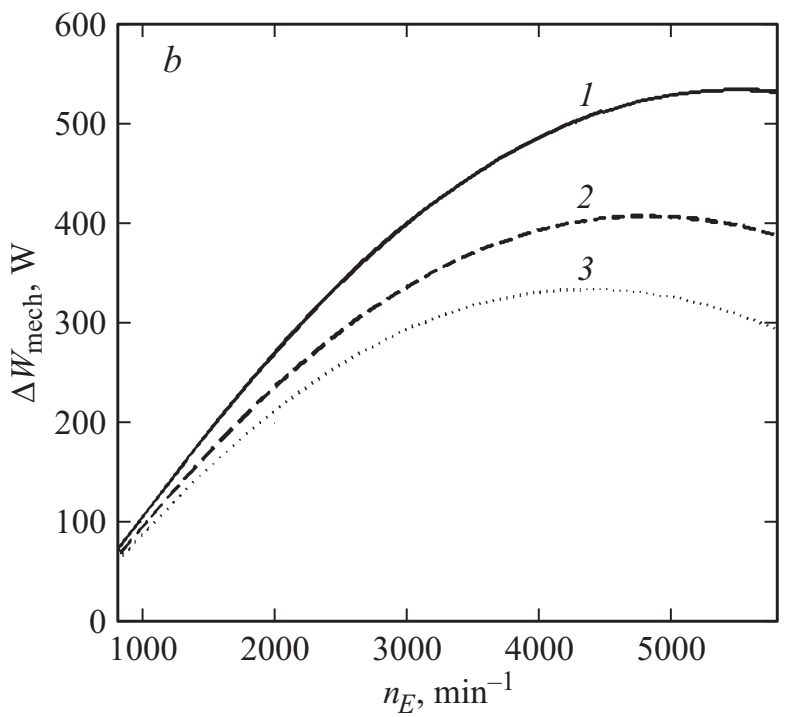

Рис. 5. Влияние контактных тепловых сопротивлений на мощности ТЭБ $W_{\text {teb }}(a)$ и на увеличение мощности ДВС $\Delta W_{\text {mech }}(b)$ : $1-R_{\text {cont }}=0,2-$ холодный контакт с термопастой, горячий контакт с эмалью, $3-$ чистая полированная поверхность контакта, - - эксперимент. 
помощью тепловизора на стенде для измерения термоэлектрических полупроводниковых батарей, собранном на основе пресса MEGA KSC-15. Из рис. 5 видно, что применение термопасты и эмали позволяют повысить электрическую мощность на $\sim 10 \%$ по сравнению с сухим шлифованным стыком, но дальнейшее снижение контактных сопротивлений может дать ограниченный результат, поскольку $W_{\text {teb }}$ уже приближается к предельному значению при $R_{\text {cont }}=0$.

Полученное расчетное значение $W_{\text {teb }}$ оказалось ниже на 7\% экспериментально полученного значения $W_{\text {teb }}=439$ Вт на макете ТЭГ.

\section{6. Заключение и результаты}

Разработана модель для численного расчета автомобильных ТЭГ с учетом обратного влияния на ДВС, обусловленного дополнительным гидравлическим сопротивлением, пригодная для параметрической оптимизации.

Достигнутые значения мощности термоэлектрического $W_{\text {teb }}$ свидетельствуют о потенциальной возможности замены штатного генератора автомобиля. Но для этого необходимо провести оптимизацию конструкции с учетом стоимости, веса и динамических процессов при смене режимов автомобиля.

Полученные результаты свидетельствуют о том, что при проектировании ТЭГ для автомобиля необходимо учитывать его обратное влияние на ДВС из-за значительного гидравлического сопротивления, которое создают конструкции ТЭГ с развитым оребрением и интенсификаторами ОГ.

Преодоление конфликта между положительным и негативным влиянием ТЭГ на ДВС возможно за счет рационального проектирования конструкции теплообменника, или применения теплообменника, у которого геометрия ребер могла бы быть изменяемой для снижения сопротивления при больших скоростях ОГ и повышения теплового потока при малых скоростях.

Показан ограниченный положительный эффект от снижения контактного теплового сопротивления за счет применения эмали и термопасты.

Расчетные значения электрической мощности свидетельствуют о потенциальной возможности замены штатного автомобильного генератора. Экспериментально полученное значение $W_{\text {teb }}=439$ Вт подтверждает этот вывод.

Ведущиеся разработки полупроводниковых материалов с увеличенным значением критерия Иоффе $Z T>0.5-0.9$ позволит существенно повысить производительность автомобильных ТЭГ, или уменьшить их массу и габариты.

Работа выполнена при финансовой поддержке Министерства образования и науки России (грант 14.577.21.0113) идентификационный номер RFMEFI57714X0113.

\section{Список литературы}

[1] L. Weiling, T. Shantung. Chin. Sci. Bull., 49, 1212 (2004).

[2] Y. Wang, S. Li, X. Yang. J. Electron. Mater., 45, 1792 (2016).

[3] Х.М. Сакр, М.X. Мансур, М.Н. Мусса. Термоэлектричество, 1, 64 (2008).

[4] С.А. Варламов, А.С. Иванов, Ю.П. Прилепо. Энергетика. Изв. акад. наук, 3, 90 (2011).

[5] C.Q. Su, C. Huang, Y.D. Deng. J. Electron. Mater., 45, 1464 (2016).

[6] M. MusiaE, M. Borcuch, K. Wojciechowski. J. Electron. Mater., 45 (3), 1517 (2016).

[7] S. Kumar, S.D. Heister, X. Xu, J.R. Salvador, G.P. Meisner. J. Electron. Mater., 42 (4), 665 (2013).

[8] М.А. Коржуев. Письма ЖТФ, 37 (4), 8 (2011).

[9] X. Gou, H. Xiao, S. Yang. Appl. Energy, 87 (10), 3131 (2010).

[10] А. Леонтьев, Д. Онищенко, Г. Арутюнян. Теплофизика и аэромеханика, 23, 747 (2016).

[11] R.A. Poshekhonov, A.S. Osipkov, M.O. Makeev. Global J. Pure Appl. Mathematics, 12 (1), 677 (2016).

[12] Программный комплекс Дизель-РК. http://www.dieselrk.bmstu.ru

[13] J.S. Jadhao, D.G. Thombare. J. Engineering Innovative Technol., 12, 93 (2013).

[14] В.П. Исаченко, В.А. Осипова, А.С. Сукомел. Теплопередача. Учебник для вузов (М., Энергия, 1975).

Редактор Г.А. Оганесян

\section{Mathematical model for optimization of the automotive thermoelectric generator structure, considering the influence of its hydraulic resistance at the engine power}

\section{R.A. Poshekhonov, G.A. Arutyunyan, S.A. Pankratov, A.S. Osipkov, D.O. Onishchenko, A.I. Leontyev \\ Bauman Moscow State Technical University, 105005 Moscow, Russia}

\begin{abstract}
An approach and a range of models designed for optimization structure and operational modes of automotive thermoelectric generator, taking into account the impact of its hydraulic resistance to the internal combustion engine are proposed. A series of thermoelectric generators that convert thermal energy of exhaust gases into electricity due to the Seebeck effect in the semiconductor elements with different geometry of the hot heat exchangers and different flow resistance were calculated.

Models for the calculation of thermoelectric elements, gas heat exchanger and an automobile engine were together considered. The simulation of the thermoelectric generator for the engine VAZ 21126 have shown the possibility of obtaining up to 500 watts of electrical power by sing semiconductor thermoelectric elements based on germanium telluride, and lead.
\end{abstract}

